\title{
Deformed Gauge Invariance with Massive Gauge Vector Bosons
}

\author{
Dao Vong Duc ${ }^{1}$, Nguyen Mong Giao², Tran Thanh Dung3 \\ ${ }^{1}$ Institute of Physics, Hanoi, Vietnam \\ ${ }^{2}$ Center for Nuclear Research, HCM City, Vietnam \\ ${ }^{3}$ Thu Dau Mot University, Binh Duong Province, Vietnam \\ Email: dvduc@iop.vast.vn,nmgiao2011@yahoo.com.vn, ttdung24@gmail.com
}

How to cite this paper: Duc, D.V., Giao, N.M. and Dung, T.T. (2017) Deformed Gauge Invariance with Massive Gauge Vector Bosons. Journal of Modern Physics, 8, 82-86. http://dx.doi.org/10.4236/jmp.2017.81007

Received: November 3, 2016

Accepted: January 10, 2017

Published: January 13, 2017

Copyright $\odot 2017$ by authors and Scientific Research Publishing Inc. This work is licensed under the Creative Commons Attribution International License (CC BY 4.0).

http://creativecommons.org/licenses/by/4.0/

\begin{abstract}
We consider the concept of deformed gauge invariance. The described formalism allows the vector gauge bosons to be massive independently of Higgs mechanism. It also allows the possibility for the variability of gauge coupling constants in spacetime.
\end{abstract}

\section{Keywords}

Gauge Invariance, Vector Boson Mass

\section{Introduction}

The mass problem for elementary particles in general, and for gauge vector bosons in particular, has been of actual character in many aspects, mostly in the construction of various unification models based on gauge invariance principle [1]-[7] where the Higgs mechanism for mass creation plays a crucial role.

On the other hand, in our recent works [8]-[12], a mechanism for mass creation in space-time with extradimensions has been proposed. For vector bosons, this problem has been treated in more detail in [8].

In this work, an alternative approach is proposed to give the possibility for gauge vector bosons to acquire mass independently of Higgs mechanism. It is based on a modified gauge principle and referred to as deformed gauge invariance [12].

As a consequence, the proposed mechanism also allows the possibility for gauge coupling constants to be variable in space-time. This would be meaningful for the study of both micro and macro world [13] [14].

\section{Deformed $U(1)$ Gauge Invariance}

Let $\varphi(x)$ be some matter field with $U(1)$ charge $\mathrm{q}$ and the transformation law 


$$
\varphi(x) \rightarrow \varphi^{\prime}(x)=\mathrm{e}^{-i q \omega(x)} \varphi(x)
$$

under gauge transformation with parameter $\omega(x)$.

The covariant derivative is constructed by the formula

$$
D_{\mu} \varphi(x)=\partial_{\mu} \varphi(x)-i q \mathrm{e}^{g(x)} A_{\mu}(x) \varphi(x)
$$

with the gauge field $A_{\mu}(x)$ obeying the transformation law:

$$
A_{\mu}^{\prime}(x)=A_{\mu}(x)-\mathrm{e}^{-g(x)} \partial_{\mu} \omega(x)
$$

$g(x)$ being some scalar function parameter.

The conventional field strength defined as

$$
F_{\mu v}(x) \equiv \partial_{\mu} A_{v}-\partial_{v} A_{\mu}
$$

is no more imvariant under the transformation (3) but its deformed version:

$$
F_{\mu \nu}^{(g)} \equiv F_{\mu \nu}+\partial_{\mu} g \cdot A_{\nu}-\partial_{\nu} g \cdot A_{\mu} .
$$

Hence the corresponding invariant Lagrangian should be taken of the form:

$$
\begin{aligned}
L^{(g)}(A) & \equiv-\frac{1}{4} F_{\mu \nu}^{(g)} F_{\mu \nu}^{(g)} \\
& =-\frac{1}{4} F_{\mu \nu} F^{\mu \nu}-\frac{1}{2} \partial_{\mu} g \cdot \partial^{\mu} g \cdot A_{v} A^{v}+\frac{1}{2}\left(\partial_{\mu} g \cdot A^{\mu}\right)^{2}-F_{\mu \nu} \partial^{\mu} g \cdot A^{\nu} .
\end{aligned}
$$

The Euler-Lagrange equation

$$
\frac{\delta L^{(g)}}{\delta A_{\nu}}-\partial_{\mu} \frac{\delta L^{(g)}}{\delta\left(\partial_{\mu} A_{\nu}\right)}=0
$$

then gives:

$$
\begin{aligned}
& \left(\square-\partial_{\mu} g \cdot \partial^{\mu} g+\square g\right) A_{v}+\partial_{\nu} g \cdot \partial^{\mu} g \cdot A_{\mu}+\partial^{\mu} g \cdot \partial_{\nu} A_{\mu} \\
& -\partial_{\nu} \partial^{\mu} A_{\mu}-\partial_{\nu} \partial^{\mu} g \cdot A_{\mu}-\partial_{\nu} g \cdot \partial^{\mu} A_{\mu}=0 .
\end{aligned}
$$

Let us put the constraint on the gauge field $A_{\mu}$ :

$$
\partial^{\mu} A_{\mu}=\partial^{\mu} g \cdot A_{\mu} .
$$

This coincides with the ordinary Lorentz gauge condition when $g$ is constant. Equation (8) now reads:

$$
\left(\square-\partial_{\mu} g \cdot \partial^{\mu} g+\square g\right) A_{\nu}-2 \partial_{\nu} \partial^{\mu} g \cdot A_{\mu}=0 .
$$

Now we restrict the consideration to a special form of $g(x)$, namely

$$
g(x)=a x^{2}+p x+c
$$

where $p x \equiv p_{\mu} x^{\mu}, \alpha$ and $c$ being some scalar parameters, $p_{\mu}$-some vector parameter.

With Equation (11) inserted Equation (10) becomes:

$$
\left\{\square-p^{2}+4 a\left(1-p x-a x^{2}\right)\right\} A_{\mu}(x)=0
$$

which corresponds to the expression

$$
m_{A}^{2}=-p^{2}+4 a\left(1-p x-a x^{2}\right)
$$


for mass of gauge boson $A$.

Equation (13) shows that in general $m_{A}$ can vary in value in space-time, except for the special case $a=0$, where $m_{A}^{2}=-p^{2}$. It takes the value

$$
m_{A}^{2}=4 a-p^{2}
$$

at the origin $x_{\mu}=0$.

\section{Deformed Non-Abelian Gauge Invariance}

We now proceed to the case of non-abelian gauge. Let $\{\varphi(x)\}$ be some matter field multiplet obeying the transformation law under gauge transformation

$$
\begin{gathered}
\varphi_{i}(x) \rightarrow \varphi_{i}^{\prime}(x)=(S(x))_{i}^{j} \varphi_{j}(x) \\
S(x) \equiv \mathrm{e}^{-i \sum_{a} \omega_{a}(x) M_{a}}
\end{gathered}
$$

$M_{a}$ being representation matrices of the symmetry algebra

$$
\left[M_{a}, M_{b}\right]=i f_{a b c} M_{c}
$$

$f_{a b c}$-structure constants.

The covariant derivative is introduced by the formula:

$$
\begin{gathered}
D_{\mu} \varphi_{i}(x)=\partial_{\mu} \varphi_{i}(x)-i G \mathrm{e}^{g(x)}\left(A_{\mu}\right)_{i}^{j} \varphi_{j}(x) \\
A_{\mu} \equiv \sum_{a} A_{\mu a} M_{a}
\end{gathered}
$$

with the gauge fields $A_{\mu a}(x)$ transforming according to the rule:

$$
A_{\mu}^{\prime}(x)=S A_{\mu} S^{-1}+\frac{i}{G} \mathrm{e}^{-g(x)} S \cdot \partial_{\mu} S^{-1}
$$

$G$ being gauge coupling constant.

The deformed field strength $F_{\mu v a}^{(g)}$ is constructed from the conventional one

$$
F_{\mu v a} \equiv \partial_{\mu} A_{v a}-\partial_{\nu} A_{\mu a}
$$

in a similar way as Equation (5), namely:

$$
F_{\mu v a}^{(g)} \equiv F_{\mu v a}+\partial_{\mu} g \cdot A_{v a}-\partial_{\nu} g \cdot A_{\mu a}+G \mathrm{e}^{g(x)} \sum_{b, c} f_{a b c} A_{\mu b} A_{v c}
$$

with the transformation law:

$$
\begin{gathered}
F_{\mu \nu}^{(g)^{\prime}}=S F_{\mu \nu}^{(g)} S^{-1} \\
F_{\mu \nu}^{(g)} \equiv \sum_{a} F_{\mu \nu a}^{(g)} M_{a} .
\end{gathered}
$$

Hence, the invariant Lagrangian for gauge fields should be:

$$
\begin{aligned}
L^{(g)}(A)= & -\frac{1}{2} \operatorname{Tr} F_{\mu \nu}^{(g)} F^{(g) \mu \nu} \\
= & -\frac{1}{4} F_{\mu v a} F_{a}^{\mu \nu}-\frac{1}{2} \partial_{\mu} g \cdot \partial^{\mu} g \cdot A_{v a} A_{a}^{v} \\
& +\frac{1}{2}\left(\partial_{\mu} g \cdot A_{a}^{\mu}\right) \cdot\left(\partial_{\nu g} \cdot A_{a}^{v}\right)-F_{\mu v a} \cdot \partial^{\mu} g \cdot A_{a}^{v}+L_{\text {int }}^{(g)}(A)
\end{aligned}
$$

where $L_{\text {int }}^{(g)}(A)$ is proportional to $G \mathrm{e}^{g}$ and $\left(G \mathrm{e}^{g}\right)^{2}$. 
By performing further calculations in a similar way as for the $U(1)$-gauge with the deformed Lorentz gauge condition

$$
\partial^{\mu} A_{\mu a}=\partial^{\mu} g \cdot A_{\mu a}
$$

taken into account the same expression (13) for mass $\mathrm{mA}$ will be obtained.

\section{Variable Coupling Constants}

From the Equations (2) and (17) of covariant derivatives it follows immediately that instead of the gauge coupling constants $q$ and $G$ one should use

$$
\tilde{q}(x) \equiv q \cdot \mathrm{e}^{g(x)} \quad \tilde{G}(x) \equiv G \cdot \mathrm{e}^{g(x)}
$$

in the corresponding gauge interaction Lagrangians instead of $q$ and $G$.

For example, the $U(1)$-gauge interaction Lagrangians for charged scalar field $\phi(x)$ and spinor field $\psi(x)$ should be:

$$
\begin{gathered}
L_{\text {int }}(\phi, A)=q \mathrm{e}^{g(x)} \cdot \phi^{+}(x) \vec{\partial}_{\mu} \phi(x) \cdot A^{\mu}(x)+q^{2} \mathrm{e}^{2 g(x)} \phi^{+}(x) \phi(x) A^{\mu}(x) A_{\mu}(x) \\
L_{\text {int }}(\psi, A)=q \mathrm{e}^{g(x)} \cdot \bar{\psi}(x) \gamma_{\mu} \psi(x) \cdot A^{\mu}(x)
\end{gathered}
$$

respectively.

Hence, according to the formalism presented here the fine structure constant $\alpha$ can change the value in space-time. In this connection it is worth mentioning that the problem concerning the variability of $\alpha$ is significant for the study of both macro and micro world. In fact, is has been realized that if so, many phenomena of the Nature related to the time evolution of the Universe might be theoretically explained. Take for example the Red Shift in cosmology traditionally treated as Doppler effect. Within our proposed mechanism, it might be explained in an alternative way more compatible with static Universe in General Relativity. Another example would be the Oklo problem [13] [14] which might be theoretically explained if the value of $\alpha$ some milliards years ago was far different from that at present time.

The variability of coupling constants in space-time might also have the relation to the renormalization problem in quantum field theory, this topic is the subject of our further consideration.

\section{Conclusion}

In this work, the concept of deformed gauge is considered. The key idea is the introduction of some parameter function $g(x)$ in the transformation law for gauge fields. The proposed formalism might be considered as the generalization of the traditional gauge invariance which corresponds to the special case $g(x)=0$. The formalism allows the gauge vector bosons to acquire mass with the value expressed in terms of $g(x)$. It also allows the possibility for the gauge coupling constant to be variable in space-time.

\section{References}

[1] Furlan, G., Jengo, R., Pati, J.C., Sciama, D.W. and Shafi, O. (1997) Superstrings, Supergravity and Unified Theories. World Scientific, Singapore City. 
[2] Konuma, M. and Maskawa, T. (1981) Grand Unified Theories and Related Topics. World Scientific, Singapore City.

[3] Zee, A. (1985) Unity of Forces in the Universe. World Scientific, Singapore City.

[4] Becker, K., Becker, M. and Schwarz, J.H. (2007) String Theory and M-Theory.

[5] Green, M.B. and Gross, D.J. (1981) Unified Field Theory. World Scientific, Singapore City.

[6] O’Raifeartaigh, L. and Straumann, N. (1997) Earky History of Gauge Theories and Kaluza-Klein Theories. arXiv-helth /98/05.

[7] Moriyasu, K. (1985) An Elementary Primer for Gauge Theory. World Scientific, Singapore City.

[8] Duc, D.V. and Giao, N.M. (2013) Journal of Modern Physics, 4, 991. https://doi.org/10.4236/jmp.2013.47133

[9] Duc, D.V. and Giao, N.M. (2014) Journal of Modern Physics, 5, 477. https://doi.org/10.4236/jmp.2014.56058

[10] Duc, D.V., Giao, N.M. and Dung, T.T. (2015) International Journal of Theoretical Physics, 54, 1071. https://doi.org/10.1007/s10773-014-2300-9

[11] Duc, D.V. and Giao, N.M. (2016) International Journal of Theoretical Physics, 55, 959. https://doi.org/10.1007/s10773-015-2740-x

[12] Duc, D.V. (2011) Communications in Physics (Vietnam), 21, 259.

[13] Yasumori, F. (2004) Oklo Constraint on the Time Variability of Fine Structure Constant, Astrophysics, Clocks and Fundamental Constants. Lecture Notes in Physics. Springer Berlin, 167-185.

[14] Lamoreaux, S.K. and Torgeson, J.R. (2004) Physical Review D, 69, 1217016. https://doi.org/10.1103/PhysRevD.69.121701

\section{Submit or recommend next manuscript to SCIRP and we will provide best service} for you:

Accepting pre-submission inquiries through Email, Facebook, LinkedIn, Twitter, etc. A wide selection of journals (inclusive of 9 subjects, more than 200 journals)

Providing 24-hour high-quality service

User-friendly online submission system

Fair and swift peer-review system

Efficient typesetting and proofreading procedure

Display of the result of downloads and visits, as well as the number of cited articles

Maximum dissemination of your research work

Submit your manuscript at: http://papersubmission.scirp.org/

Or contact jmp@scirp.org 